IJMMS 29:9 (2002) 545-553

PII. S0161171202007858

http://ijmms.hindawi.com

(c) Hindawi Publishing Corp.

\title{
ON A CLASS OF DIOPHANTINE EQUATIONS
}

\author{
SAFWAN AKBIK
}

Received 10 June 2001

Cohn (1971) has shown that the only solution in positive integers of the equation $Y(Y+$ $1)(Y+2)(Y+3)=2 X(X+1)(X+2)(X+3)$ is $X=4, Y=5$. Using this result, Jeyaratnam (1975) has shown that the equation $Y(Y+m)(Y+2 m)(Y+3 m)=2 X(X+m)(X+2 m)(X+$ $3 m$ ) has only four pairs of nontrivial solutions in integers given by $X=4 m$ or $-7 m$, $Y=5 m$ or $-8 m$ provided that $m$ is of a specified type. In this paper, we show that if $m=\left(m_{1}, m_{2}\right)$ has a specific form then the nontrivial solutions of the equation $Y(Y+$ $\left.m_{1}\right)\left(Y+m_{2}\right)\left(Y+m_{1}+m_{2}\right)=2 X\left(X+m_{1}\right)\left(X+m_{2}\right)\left(X+m_{1}+m_{2}\right)$ are $m$ times the primitive solutions of a similar equation with smaller $m$ 's. Then we specifically find all solutions in integers of the equation in the special case $m_{2}=3 m_{1}$.

2000 Mathematics Subject Classification: 11D25, 11D45, 11D09, 11D41.

We generalize the equations of Cohn [1] and Jeyaratnam [2] by considering the Diophantine equation

$$
Y\left(Y+m_{1}\right)\left(Y+m_{2}\right)\left(Y+m_{1}+m_{2}\right)=2 X\left(X+m_{1}\right)\left(X+m_{2}\right)\left(X+m_{1}+m_{2}\right) .
$$

The trivial solutions of (1) are the sixteen pairs obtained by equating both sides of the equation to zero. A nontrivial solution with $\left(X, Y, m_{1}, m_{2}\right)=1$ is called a primitive solution.

THEOREM 1. If every prime $p$ dividing $m=\left(m_{1}, m_{2}\right)$ is such that

$$
p \equiv 2,3,5(\bmod 8) \quad \text { or } \quad p \equiv 1(\bmod 8) \quad \text { with } 2^{(p-1) / 4} \equiv-1(\bmod p),
$$

then every nontrivial solution of (1) is $m$ times a primitive solution of

$$
Y\left(Y+\frac{m_{1}}{m}\right)\left(Y+\frac{m_{2}}{m}\right)\left(Y+\frac{m_{1}+m_{2}}{m}\right)=2 X\left(X+\frac{m_{1}}{m}\right)\left(X+\frac{m_{2}}{m}\right)\left(X+\frac{m_{1}+m_{2}}{m}\right) .
$$

THEOREM 2. If every prime $p$ dividing $N$ is of the form (2), then every nontrivial solution of

$$
Y(Y+N)(Y+c N)(Y+(1+c) N)=2 X(X+N)(X+c N)(X+(1+c) N)
$$

is $N$ times a nontrivial solution of

$$
Y(Y+1)(Y+c)(Y+1+c)=2 X(X+1)(X+c)(X+1+c),
$$

where $c$ is a positive integer. 
THEOREM 3. The equation

$$
Y(Y+1)(Y+3)(Y+4)=2 X(X+1)(X+3)(X+4)
$$

has only four pairs of nontrivial solutions in integers given by $X=14$ or $-18, Y=17$ or -21 .

THEOREM 4. If every prime $p$ dividing $N$ is of the form (2), then the equation

$$
Y(Y+N)(Y+3 N)(Y+4 N)=2 X(X+N)(X+3 N)(X+4 N)
$$

has only four pairs of nontrivial solutions in integers given by $X=14 \mathrm{~N}$ or $-18 \mathrm{~N}$, $Y=17 N$ or $-21 N$.

Note that Theorem 2 follows immediately by applying Theorem 1 with $m_{1}=N, m_{2}=$ $c N$, and $m=(N, c N)=N$. Also Theorem 4 follows easily by combining Theorem 2 , in the case $c=3$, with Theorem 3 .

LEMMA 5. Every solution of (1) that is not primitive is $K=\left(X, Y, m_{1}, m_{2}\right)$ times a primitive solution of

$$
Y\left(Y+\frac{m_{1}}{K}\right)\left(Y+\frac{m_{2}}{K}\right)\left(Y+\frac{m_{1}+m_{2}}{K}\right)=2 X\left(X+\frac{m_{1}}{K}\right)\left(X+\frac{m_{2}}{K}\right)\left(X+\frac{m_{1}+m_{2}}{K}\right) .
$$

Proof. Suppose that $X, Y$ is a solution of (1). By dividing both sides of that equation by $K^{4}$ we find

$$
\begin{aligned}
\frac{Y}{K}\left(\frac{Y}{K}+\frac{m_{1}}{K}\right) & \left(\frac{Y}{K}+\frac{m_{2}}{K}\right)\left(\frac{Y}{K}+\frac{m_{1}+m_{2}}{K}\right) \\
& =2 \cdot \frac{X}{K}\left(\frac{X}{K}+\frac{m_{1}}{K}\right)\left(\frac{X}{K}+\frac{m_{2}}{K}\right)\left(\frac{X}{K}+\frac{m_{1}+m_{2}}{K}\right) .
\end{aligned}
$$

Thus $X / K, Y / K$ is a solution of (8). The lemma follows since $\left(X / K, Y / K, m_{1} / K\right.$, $\left.m_{2} / K\right)=1$.

LEMMA 6. Equation (1) cannot have a primitive solution if the greatest common divisor $m=\left(m_{1}, m_{2}\right)$ is divisible by a prime $p$ of the form (2).

Proof. By completing the squares in (1) we find

$$
\left[\frac{\left(2 Y+m_{1}+m_{2}\right)^{2}-m_{1}^{2}-m_{2}^{2}}{2}\right]^{2}-2\left[\frac{\left(2 X+m_{1}+m_{2}\right)^{2}-m_{1}^{2}-m_{2}^{2}}{2}\right]^{2}=-m_{1}^{2} m_{2}^{2} \text {. }
$$

Letting

$$
\begin{gathered}
y=2 Y+m_{1}+m_{2}, \\
x=2 X+m_{1}+m_{2}, \\
A=\frac{y^{2}-m_{1}^{2}-m_{2}^{2}}{2}=2 Y^{2}+2 Y\left(m_{1}+m_{2}\right)+m_{1} m_{2}, \\
B=\frac{x^{2}-m_{1}^{2}-m_{2}^{2}}{2}=2 X^{2}+2 X\left(m_{1}+m_{2}\right)+m_{1} m_{2},
\end{gathered}
$$


we obtain the equations

$$
\begin{gathered}
y^{2}=2 A+m_{1}^{2}+m_{2}^{2}, \quad x^{2}=2 B+m_{1}^{2}+m_{2}^{2}, \\
A^{2}-2 B^{2}=-m_{1}^{2} m_{2}^{2} .
\end{gathered}
$$

If $2 \mid m$, then

$$
\begin{aligned}
A^{2}-2 B^{2}=-m_{1}^{2} m_{2}^{2} & \Longrightarrow A, B \equiv 0(\bmod 4) \underset{\text { by }(13)}{\Longrightarrow} 2 X^{2}, 2 Y^{2} \equiv 0(\bmod 4) \\
& \Longrightarrow X, Y \equiv 0(\bmod 2) \Longrightarrow 2 \mid\left(X, Y, m_{1}, m_{2}\right) \neq 1 .
\end{aligned}
$$

Let $p \mid m$ such that $p \equiv 3,5(\bmod 8)$. Assume that $p \nmid A$, then by (15), $p \nmid B$. Also by (15), $1=\left(2 B^{2} / p\right)=(2 / p)=-1$, a contradiction. Thus $p \mid A$ and hence $p \mid B$. By (13), $p \mid X$ and $Y$. Therefore $\left(X, Y, m_{1}, m_{2}\right) \neq 1$.

Suppose that $p \mid m$ such that $p \equiv 1(\bmod 8)$ and $2^{(p-1) / 4} \equiv-1(\bmod p)$. If $p \nmid A$, then $p \nmid B$. Since $(2 / p)=1,(13)$ implies that $A$ and $B$ are quadratic residues $\bmod p$. Thus $B^{(p-1) / 2} \equiv A^{(p-1) / 2} \equiv 1(\bmod p)$. From $(15)$ we find that

$$
2 B^{2} \equiv A^{2}(\bmod p) \Longrightarrow 2^{(p-1) / 4} B^{(p-1) / 2} \equiv A^{(p-1) 2} \Longrightarrow 2^{(p-1) / 4} \equiv 1(\bmod p),
$$

a contradiction. Therefore $p \mid A, B$. By (13), $p \mid X, Y$ and hence $\left(X, Y, m_{1}, m_{2}\right) \neq 1$ and the lemmas follows.

Proof of TheOrem 1. By Lemmas 5 and 6 and the fact that $\left(m_{1} / K, m_{2} / K\right)$ can only have prime divisors of the form (2), a nontrivial solution of (2) is a multiple of a primitive solution of (3) with $\left(m_{1} / K, m_{2} / K\right)=1$. This happens when $K=\left(m_{1}, m_{2}\right)=m$ and the theorem follows.

For Theorem 3 we now prove the following lemma.

LEMMA 7. The only solution in positive integers of (6) is $X=14, Y=17$.

Proof. Note that (6) can be obtained from (1) by letting $m_{1}=1$ and $m_{2}=3$. Then (11), (12), (13), (14), and (15) become

$$
\begin{array}{cl}
y=2 Y+4, & x=2 X+4, \\
A=2 Y^{2}+8 Y+3, & B=2 X^{2}+8 X+3, \\
y^{2}=2 A+10, & x^{2}=2 B+10, \\
A^{2}-2 B^{2}=-9 .
\end{array}
$$

All solutions in positive integers of (21) are given by

$$
A=V_{n}, \quad B=U_{n},
$$

where

$$
V_{n}+\sqrt{2} U_{n}=(3+3 \sqrt{2})(3+2 \sqrt{2})^{n}=3(1+\sqrt{2})^{2 n+1}, \quad n=0,1,2, \ldots
$$


Thus

$$
\begin{aligned}
& V_{n}=\frac{3(1+\sqrt{2})^{2 n+1}+3(1-\sqrt{2})^{2 n+1}}{2}, \\
& U_{n}=\frac{3(1+\sqrt{2})^{2 n+1}-3(1-\sqrt{2})^{2 n+1}}{-2 \sqrt{2}} .
\end{aligned}
$$

Let $\alpha=1+\sqrt{2}$ and $\beta=1-\sqrt{2}$, then

$$
\begin{gathered}
\alpha+\beta=2, \quad \alpha-\beta=-2 \sqrt{2}, \quad \alpha \beta=-1, \\
V_{n}=3\left(\frac{\alpha^{2 n+1}+\beta^{2 n+1}}{\alpha+\beta}\right), \quad U_{n}=3\left(\frac{\alpha^{2 n+1}-\beta^{2 n+1}}{\alpha-\beta}\right) .
\end{gathered}
$$

From (20) and (22), we must have

$$
\begin{aligned}
& y^{2}=2 V_{n}+10, \\
& x^{2}=2 U_{n}+10 .
\end{aligned}
$$

Using (25), we can easily find that

$$
\begin{aligned}
V_{-n} & =-V_{n-1}, \\
U_{-n} & =U_{n-1}, \\
U_{n+2} & =6 U_{n+1}-U_{n}, \\
V_{n+2} & =6 V_{n+1}-V_{n} .
\end{aligned}
$$

Let

$$
\eta_{r}=\frac{\alpha^{r}+\beta^{r}}{\alpha+\beta}, \quad \xi_{r}=\frac{\alpha^{r}-\beta^{r}}{\alpha-\beta}
$$

then we easily find that

$$
\begin{gathered}
V_{n}=3 \eta_{2 n+1}, \quad U_{n}=3 \xi_{2 n+1}, \\
\xi_{2 r}=2 \xi_{r} \eta_{r}, \\
\eta_{2 r}=2 \eta_{r}^{2}+(-1)^{r+1}=4 \xi_{r}^{2}+(-1)^{r} \\
\eta_{m+n}=\eta_{m} \eta_{n}+2 \xi_{m} \xi_{n}, \\
\xi_{m+n}=\xi_{m} \eta_{n}+\xi_{n} \eta_{m} .
\end{gathered}
$$

Using relations (33), (34), (35), (36), and (37), we get

$$
\begin{aligned}
V_{n+r} & \equiv(-1)^{r+1} V_{n}\left(\bmod \eta_{r}\right), \\
V_{n+2 r} & \equiv V_{n}\left(\bmod \eta_{r}\right), \\
U_{n+r} & \equiv(-1)^{r+1} U_{n}\left(\bmod \eta_{r}\right), \\
U_{n+2 r} & \equiv U_{n}\left(\bmod \eta_{r}\right), \\
\eta_{3 r} & =\eta_{r}\left[4 \eta_{r}^{2}+3(-1)^{r+1}\right], \\
\xi_{3 r} & =\xi_{r}\left[4 \eta_{r}^{2}+(-1)^{r+1}\right] .
\end{aligned}
$$


Let

$$
\theta_{t}=\xi_{2^{t}}, \quad \phi_{t}=\eta_{2^{t}}
$$

then we get

$$
\begin{aligned}
\theta_{t+1} & =2 \theta_{t} \phi_{t}, \\
\phi_{t+1}=2 \phi_{t}^{2}-1 & =4 \theta_{t}^{2}+1=\phi_{t}^{2}+2 \theta_{t}^{2}, \\
\phi_{t}^{2} & =2 \theta_{t}^{2}+1 .
\end{aligned}
$$

Using (42), (43), and (44), we find that for $k=2^{t}$ we have

$$
\begin{aligned}
& \eta_{6 k}=\phi_{t+1}\left[4 \phi_{t+1}^{2}-3\right], \\
& \xi_{6 k}=\theta_{t+1}\left[4 \phi_{t+1}^{2}-1\right] .
\end{aligned}
$$

We will need some of the entries in Tables 1 and 2.

TABLE 1

\begin{tabular}{cll}
\hline$n$ & $U_{n}$ & $V_{n}$ \\
\hline 1 & 15 & 21 \\
3 & 507 & 717 \\
4 & 2955 & 4179 \\
11 & 675176043 & 954843117 \\
8 & 3410067 & 4822563 \\
23 & 1037608383669414483 & 1467399848617311837 \\
24 & 6047624848242867123 & 8552633080529593443 \\
\hline
\end{tabular}

TABLE 2

\begin{tabular}{cl}
\hline$k$ & $\eta_{k}$ \\
\hline 2 & 3 \\
3 & 7 \\
4 & 17 \\
6 & $3^{2} \cdot 11$ \\
8 & 577 \\
12 & $17 \cdot 1153$ \\
24 & $97 \cdot 577 \cdot 13729$ \\
48 & $193 \cdot 9188923201 \cdot 665857$ \\
\hline
\end{tabular}

Now we consider the following cases.

(a) Equation (26) is impossible if $n \equiv 1(\bmod 3)$. Let $n=1+3 r$ where $r \geq 0$, then using (38) we get

$$
\begin{aligned}
& V_{n} \equiv \pm V_{1}\left(\bmod \eta_{3}\right), \\
& V_{n} \equiv \pm 21 \equiv 0(\bmod 7) .
\end{aligned}
$$

Hence $2 V_{n}+10 \equiv 10 \equiv 3(\bmod 7)$. Since $(3 / 7)=-1,(26)$ is impossible. 
(b) Equation (27) is impossible if $n \equiv 1,2(\bmod 4)$. Using $(40)$, we get

$$
\begin{aligned}
& U_{n} \equiv \pm U_{1}, \pm U_{2}\left(\bmod \eta_{4}\right), \\
& U_{n} \equiv \pm 15, \pm 87 \equiv \pm 2(\bmod 17) .
\end{aligned}
$$

Hence $2 U_{n}+10 \equiv \pm 4+10 \equiv 6,-3(\bmod 17)$. Since $(6 / 17)=(-3 / 17)=-1,(27)$ is impossible.

(c) Equation (26) is impossible if $n \equiv 8(\bmod 12)$. Using (39) and (28) we get

$$
\begin{aligned}
& V_{n} \equiv V_{-4}=-V_{3}\left(\bmod \eta_{6}\right), \\
& V_{n} \equiv-717 \equiv-2(\bmod 11) \quad \text { since } 11 \mid \eta_{6} .
\end{aligned}
$$

Hence $2 V_{n}+10 \equiv 6(\bmod 11)$. Since $(6 / 11)=-1,(26)$ is impossible.

(d) Equation (26) is impossible if $n \equiv 11(\bmod 16)$. Using (39) and (28) we get

$$
\begin{aligned}
& V_{n} \equiv V_{-5}=-V_{4}\left(\bmod \eta_{8}\right), \\
& V_{n} \equiv-4179 \equiv-140(\bmod 577) .
\end{aligned}
$$

Hence $2 V_{n}+10 \equiv-270(\bmod 577)$. Since $(-270 / 577)=-1$, (26) is impossible.

(e) Equation (26) is impossible if $n \equiv 11,12(\bmod 24)$. Using (38) and (28) we get

$$
\begin{aligned}
& V_{n} \equiv \pm V_{11}, \pm V_{-12}= \pm V_{11}, \mp V_{11}\left(\bmod \eta_{24}\right), \\
& V_{n} \equiv \pm 954843117 \equiv \pm 46(\bmod 97) \quad \text { since } 97 \mid \eta_{24} .
\end{aligned}
$$

Hence $2 V_{n}+10 \equiv \pm 102+10 \equiv 5,15(\bmod 97)$. Since $(5 / 97)=(15 / 97)=-1,(26)$ is impossible.

(f) Equation (26) is impossible if $n \equiv 15(\bmod 24)$. Using (38) and (28) we get

$$
\begin{aligned}
& V_{n} \equiv \pm V_{-9}=\mp V_{8}\left(\bmod \eta_{24}\right), \\
& V_{n} \equiv \mp 4822563 \equiv \pm 504289(\bmod 1331713) \quad \text { since } 1331713 \mid \eta_{24} .
\end{aligned}
$$

Hence $2 V_{n}+10 \equiv 323145,1008588(\bmod 1331713)$. Since $(323145 / 1331713)=$ $(1008588 / 1331713)=-1,(26)$ is impossible.

(g) Equation (26) is impossible if $n \equiv 23,24(\bmod 48)$. Using (38) and (28) we get

$$
V_{n} \equiv \pm V_{23}, \pm V_{-24}= \pm V_{23}, \mp V_{23}\left(\bmod \eta_{48}\right) .
$$

Since $V_{23}=1467399848617311837$ and $\tau=9188923201 \mid \eta_{48}$, we have $2 V_{n}+10 \equiv$ $11299978,-11299958(\bmod \tau)$. Since $(11299978 / \tau)=(-11299958 / \tau)=-1$, (26) is impossible.

(h) Equation (27) is impossible if $n \equiv 3(\bmod 48), n \neq 3$. That is, $n=3+3 \cdot 2^{t}$. $r$, where $t \geq 4$ and $r$ is an odd positive integer. Using (40) we get $U_{n} \equiv-U_{3}=$ $-507\left(\bmod \eta_{3 \cdot 2^{t}}\right)$. Hence

$$
2 U_{n}+10 \equiv-1004\left(\bmod \eta_{3 \cdot 2^{t}}\right) .
$$

From (48) we get $\eta_{3 \cdot 2^{t}}=\eta_{6 \cdot 2^{t-1}}=\phi_{t}\left[4 \phi_{t}^{2}-3\right]$. Using this in (57) we simultaneously get

$$
\begin{aligned}
& 2 U_{n}+10=-1004\left(\bmod \phi_{t}\right), \\
& 2 U_{n}+10=-1004\left(\bmod 4 \phi_{t}^{2}-3\right) .
\end{aligned}
$$


Since $\phi_{t+1}=2 \phi_{t}^{2}-1$ and $\phi_{3}=577$ we can easily show, by induction, the following for $t \geq 3$

$$
\begin{aligned}
& \phi_{t} \equiv 1(\bmod 8), \\
& \phi_{t} \equiv 81,69,-17,75,-46,-36(\bmod 251),
\end{aligned}
$$

when

$$
t \equiv 0,1,2,3,4,5(\bmod 6)
$$

respectively. By (59) we get

$$
\left(\frac{-1004}{\phi_{t}}\right)=\left(\frac{-1}{\phi_{t}}\right)\left(\frac{4}{\phi_{t}}\right)\left(\frac{251}{\phi_{t}}\right)=(1)(1)\left(\frac{\phi_{t}}{251}\right)=\left(\frac{\phi_{t}}{251}\right) .
$$

Similarly $\left(-1004 /\left(4 \phi_{t}^{2}-3\right)\right)=\left(\left(4 \phi_{t}^{2}-3\right) / 251\right)$. Using $(60)$ we find that $\left(\phi_{t} / 251\right)=-1$ if $t \equiv 2,5(\bmod 6)$ and $\left(\left(4 \phi_{t}^{2}-3\right) / 251\right)=-1$ if $t \equiv 0,1,3,4(\bmod 6)$. Therefore $(27)$ is always impossible in this case.

Note that for $n=3$ we have $U_{3}=507$ and $V_{3}=717$. Now (22) and (19) imply that $X=14, Y=17$, a nontrivial solution of (6).

(i) Equation (27) is impossible if $n \equiv \delta(\bmod 48)$ and $n>0$, where $\delta=0,-1$. That is $n=\delta+3 k(2 r+1)=\delta+6 k r+3 k$, where $k=2^{t}, t \geq 4$, and $r \geq 0$. Using (40) and (33) we get

$$
U_{n} \equiv \pm U_{3 k+\delta}= \pm 3 \xi_{6 k+2 \delta+1}\left(\bmod \eta_{6 k}\right)
$$

The upper and the lower signs depend on whether $r$ is even or odd. Using (37), we get

$$
\xi_{6 k+2 \delta+1}=\xi_{6 k} \eta_{2 \delta+1}+\xi_{2 \delta+1} \eta_{6 k},
$$

where $\eta_{2 \delta+1}=1,-1$ for $\delta=0,-1$ and $\xi_{2 \delta+1}=1$ for $\delta=0,1$. Now (64) becomes $\xi_{6 k+2 \delta+1}= \pm \xi_{6 k}+\eta_{6 k}$, where the upper and lower signs depend on whether $\delta=0$ or $\delta=1$, respectively. Using this in (63) we get

$$
U_{n} \equiv \pm 3 \xi_{6 k}\left(\bmod \eta_{6 k}\right) .
$$

For $\delta=0$, the upper sign holds if $r$ is even and the lower sign holds if $r$ is odd. For $\delta=-1$, upper sign holds if $r$ is odd and the lower sign holds if $r$ is even. Using (48) and (49) in (65) we get

$$
U_{n} \equiv \pm 3 \theta_{t+1}\left[4 \phi_{t+1}^{2}-1\right]= \pm 3 \theta_{t+1}\left[4 \phi_{t+1}^{2}-3+2\right]\left(\bmod \phi_{t+1}\left[4 \phi_{t+1}^{2}-3\right]\right) .
$$

Therefore we simultaneously get $U_{n} \equiv \pm 6 \theta_{t+1}\left(\bmod 4 \phi_{t+1}^{2}-3\right)$ and $U_{n} \equiv \mp 3 \theta_{t+1}$ $\left(\bmod \phi_{t+1}\right)$. Thus

$$
\begin{aligned}
& 2 U_{n}+10 \equiv 10 \pm 12 \theta_{t+1}\left(\bmod 4 \phi_{t+1}^{2}-3\right), \\
& 2 U_{n}+10 \equiv 10 \mp 6 \theta_{t+1}\left(\bmod \phi_{t+1}\right) .
\end{aligned}
$$

In what follows we need the fact that

$$
\theta_{t} \equiv 0(\bmod 8), \quad \text { for } t \geq 3,
$$


which follows by induction using (45) and $\theta_{3}=408$. Now we show that

$$
\begin{aligned}
\left(\frac{10 \pm 12 \theta_{t+1}}{4 \phi_{t+1}^{2}-3}\right) & =\left(\frac{5 \pm 6 \theta_{t+1}}{59}\right), \\
\left(\frac{10 \mp 6 \theta_{t+1}}{\phi_{t+1}}\right) & = \pm\left(\frac{10 \theta_{t} \pm 3 \phi_{t}}{59}\right) .
\end{aligned}
$$

For (69) we have

$$
\begin{aligned}
\left(\frac{10 \pm 12 \theta_{t+1}}{4 \phi_{t+1}^{2}-3}\right) & =\left(\frac{2}{4 \phi_{t+1}^{2}-3}\right)\left(\frac{5 \pm 6 \theta_{t+1}}{4 \phi_{t+1}^{2}-3}\right) \\
& =\left(\frac{5 \pm 6 \theta_{t+1}}{4 \phi_{t+1}^{2}-3}\right), \quad \text { using (59) } \\
& =\left(\frac{5 \pm 6 \theta_{t+1}}{8 \theta_{t+1}^{2}+1}\right), \quad \text { using }(47) \\
& =\left(\frac{8 \theta_{t+1}^{2}+1}{5 \pm 6 \theta_{t+1}}\right), \quad \text { since } \theta_{t} \equiv 0(\bmod 4) \\
& =\left(\frac{36\left(8 \theta_{t+1}^{2}\right.}{5 \pm 6 \theta_{t+1}}\right)=\left(\frac{236}{5 \pm 6 \theta_{t+1}}\right) \\
& =\left(\frac{59}{5 \pm 6 \theta_{t+1}}\right), \quad \text { since } 36 \theta_{t+1}^{2} \equiv 25\left(\bmod 5 \pm 6 \theta_{t+1}\right) .
\end{aligned}
$$

Equation (69) follows since $\theta_{t} \equiv 0(\bmod 4)$. For $(70)$ we have

$$
\begin{aligned}
\left(\frac{10 \mp 6 \theta_{t+1}}{\phi_{t+1}}\right) & =\left(\frac{5 \mp 3 \theta_{t+1}}{\phi_{t+1}}\right) \\
& =\left(\frac{5\left(\phi_{t}^{2}-2 \theta_{t}^{2}\right) \mp 3 \theta_{t+1}}{\phi_{t}^{2}+2 \theta_{t}^{2}}\right), \quad \text { using (46) and (47) } \\
& =\left(\frac{-20 \theta_{t}^{2} \mp 6 \theta_{t} \phi_{t}}{\phi_{t}^{2}+2 \theta_{t}^{2}}\right), \quad \text { since } \phi_{t}^{2} \equiv-2 \theta_{t}^{2}\left(\bmod \phi_{t}^{2}+2 \theta_{t}^{2}\right) \\
& =\left(\frac{-1}{\phi_{t}^{2}+2 \theta_{t}^{2}}\right)\left(\frac{2}{\phi_{t}^{2}+2 \theta_{t}^{2}}\right)\left(\frac{\theta_{t}}{\phi_{t}^{2}+2 \theta_{t}^{2}}\right)\left(\frac{10 \theta_{t} \pm 3 \phi_{t}}{\phi_{t}^{2}+2 \theta_{t}^{2}}\right) \\
& =(1)(1)(1)\left(\frac{10 \theta_{t} \pm 3 \phi_{t}}{\phi_{t}^{2}+2 \theta_{t}^{2}}\right) \\
& =\left(\frac{\phi_{t}^{2}+2 \theta_{t}^{2}}{10 \theta_{t} \pm 3 \phi_{t}}\right)=\left(\frac{9 \phi_{t}^{2}+18 \theta_{t}^{2}}{10 \theta_{t} \pm 3 \phi_{t}}\right) \\
& =\left(\frac{118 \theta_{t}^{2}}{10 \theta_{t} \pm 3 \phi_{t}}\right), \quad \text { since } 9 \phi_{t}^{2} \equiv 100 \theta_{t}^{2}\left(\bmod 10 \theta_{t} \pm 3 \phi_{t}\right) \\
& =\left(\frac{2}{10 \theta_{t} \pm 3 \phi_{t}}\right)\left(\frac{59}{10 \theta_{t} \pm 3 \phi_{t}}\right)=-\left(\frac{59}{10 \theta_{t} \pm 3 \phi_{t}}\right) .
\end{aligned}
$$

Equation (70) follows using (59) and (68). 
Since $\theta_{3}=408, \phi_{3}=577, \phi_{t+1}=2 \phi_{t}^{2}-1$, and $\theta_{t+1}=2 \theta_{t} \phi_{t}$, we can inductively show the following:

$$
\begin{aligned}
\theta_{t} & \equiv 12,5,-12,-5(\bmod 59) \quad \text { if } t \equiv 0,1,2,3(\bmod 4), \\
\phi_{t} & \equiv-17,-13(\bmod 59) \quad \text { if } t \equiv 0,1,(\bmod 2), \text { respectively. }
\end{aligned}
$$

Using (73) and taking the upper signs in (69) and (70), we get

$$
\begin{gathered}
\left(\frac{5+6 \theta_{t+1}}{59}\right)=-1 \quad \text { if } t \equiv 2,3(\bmod 4), \\
\left(\frac{10 \theta_{t}+3 \phi_{t}}{59}\right)=-1 \quad \text { if } t \equiv 0,1,2(\bmod 4) .
\end{gathered}
$$

Thus this case is always impossible. Using the lower signs in (69) and (70) we get

$$
\begin{aligned}
\left(\frac{5-6 \theta_{t+1}}{59}\right) & =-1 \quad \text { if } t \equiv 0,1(\bmod 4), \\
-\left(\frac{10 \theta_{t}-3 \phi_{t}}{59}\right) & =-1 \quad \text { if } t \equiv 0,2,3(\bmod 4),
\end{aligned}
$$

and this case is also impossible. Therefore (27) is always impossible.

The only remaining case is $n=0$. Then $U_{0}=V_{0}=0$ and so $X=Y=0$, a trivial solution and Lemma 7 is proved.

Proof of Theorem 3. First note that if the pair $(X, Y)$ is a solution of (6), so are $(-X-4, Y),(X,-Y-4)$, and $(-X-4,-Y-4)$. Note also that $-X-4<-4$ if and only if $X>0$ and $-Y-4<-4$ if and only if $Y>0$. Since $(14,17)$ is the only solution in positive integers of $(6),(-18,17),(14,-21),(-18,-21)$ are the only solutions where each of $X$ and $Y$ is either positive or less than -4 . The only remaining possibilities for more solutions are where $X$ or $Y \in\{-4,-3,-2,-1,0\}$ where there are no nontrivial solutions and the proof is completed.

Finally note that (6) has 16 trivial solutions and 4 nontrivial solutions of a total of only 20 solutions.

\section{REFERENCES}

[1] J. H. E. Cohn, The Diophantine equation $Y(Y+1)(Y+2)(Y+3)=2 X(X+1)(X+2)(X+3)$, Pacific J. Math. 37 (1971), 331-335.

[2] S. Jeyaratnam, The Diophantine equation $Y(Y+m)(Y+2 m)(Y+3 m)=2 X(X+m)(X+$ $2 m)(X+3 m)$, Pacific J. Math. 60 (1975), no. 1, 183-187.

Safwan Akbik: Department of Mathematics, Hofstra University, Hempstead, NY 11550, USA 


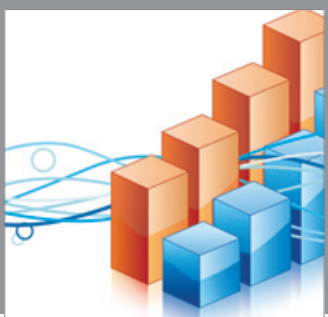

Advances in

Operations Research

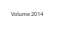

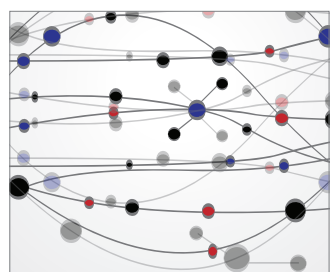

\section{The Scientific} World Journal
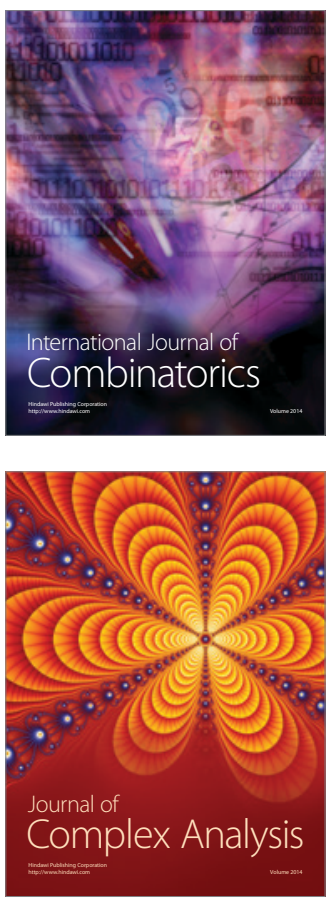

International Journal of

Mathematics and

Mathematical

Sciences
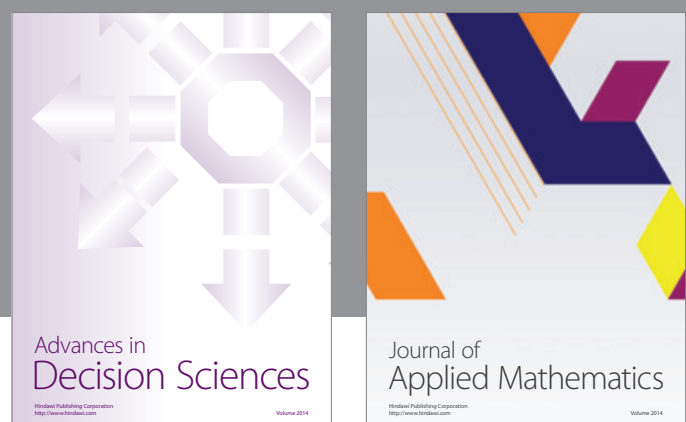

Journal of

Applied Mathematics
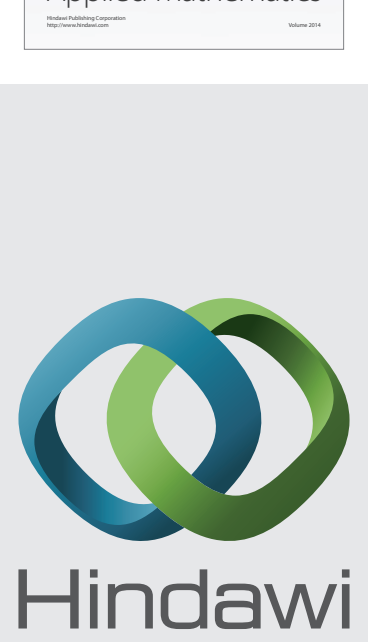

Submit your manuscripts at http://www.hindawi.com
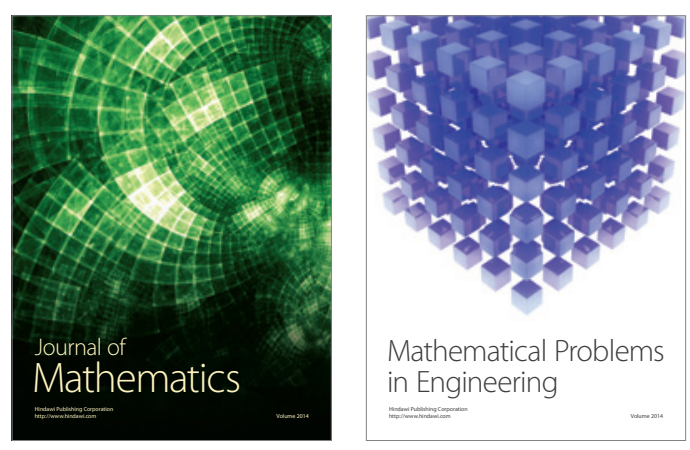

Mathematical Problems in Engineering
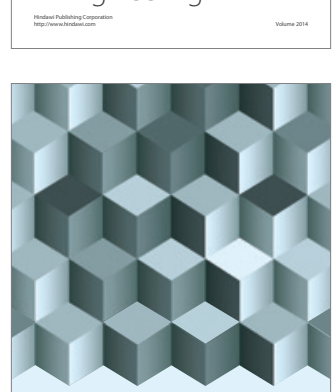

Journal of

Function Spaces
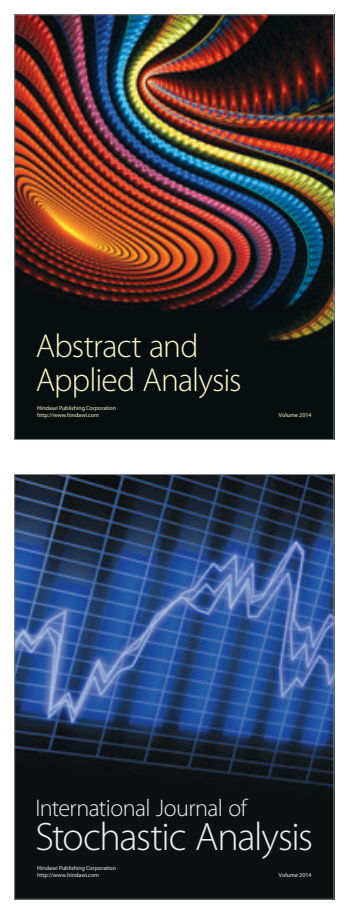

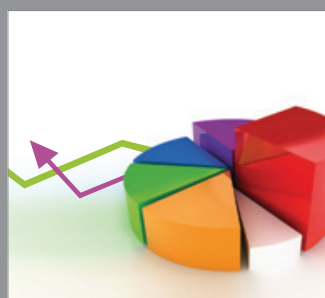

ournal of

Probability and Statistics

Promensencen
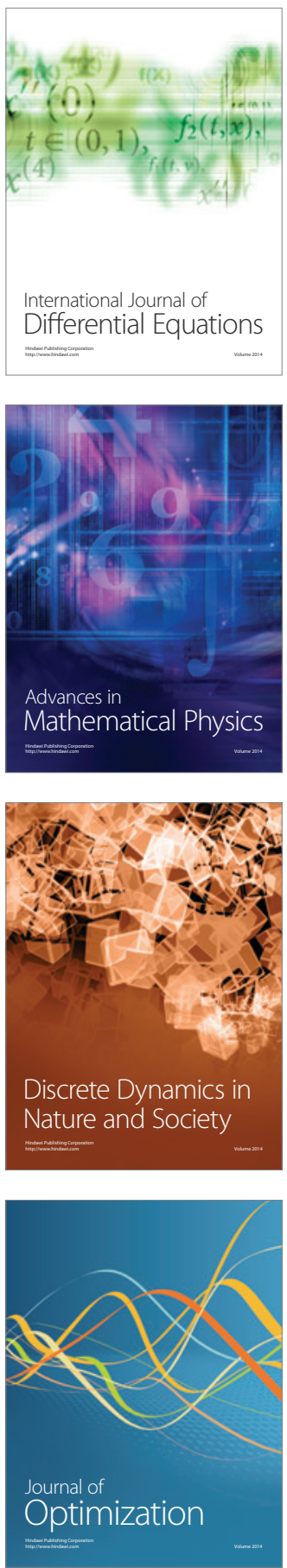